\title{
Plasmodium vivax in vitro continuous culture: the spoke in the wheel
}

\author{
Maritza Bermúdez ${ }^{1}$, Darwin Andrés Moreno-Pérez ${ }^{2,3}$, Gabriela Arévalo-Pinzón ${ }^{1}$, Hernando Curtidor 1,4 \\ and Manuel Alfonso Patarroyo $2,4^{*}$
}

\begin{abstract}
Understanding the life cycle of Plasmodium vivax is fundamental for developing strategies aimed at controlling and eliminating this parasitic species. Although advances in omic sciences and high-throughput techniques in recent years have enabled the identification and characterization of proteins which might be participating in $P$. vivax invasion of target cells, exclusive parasite tropism for invading reticulocytes has become the main obstacle in maintaining a continuous culture for this species. Such advance that would help in defining each parasite protein's function in the complex process of P. vivax invasion, in addition to evaluating new therapeutic agents, is still a dream. Advances related to maintenance, culture medium supplements and the use of different sources of reticulocytes and parasites (strains and isolates) have been made regarding the development of an in vitro culture for P. vivax; however, only some cultures having few replication cycles have been obtained to date, meaning that this parasite's maintenance goes beyond the technical components involved. Although it is still not yet clear which molecular mechanisms $P$. vivax prefers for invading young $\mathrm{CD} 71^{+}$reticulocytes [early maturation stages $(I-|I-| I \mid)$ ], changes related to membrane proteins remodelling of such cells could form part of the explanation. The most relevant aspects regarding $P$. vivax in vitro culture and host cell characteristics have been analysed in this review to explain possible reasons why the species' continuous in vitro culture is so difficult to standardize. Some alternatives for P. vivax in vitro culture have also been described.
\end{abstract}

Keywords: Plasmodium vivax, Reticulocyte, In vitro culture, Receptor, Ligand

\section{Background}

Continuous in vitro Plasmodium falciparum culture (standardized in the 1970s) [1-4] has been an indispensable tool for understanding the parasite's life cycle and identifying most proteins involved in erythrocyte invasion, some of which have been tested as vaccine components at clinical level [1-6]. Developing a methodology enabling the continuous growth and propagation of Plasmodium vivax (P. vivax being the second most important species causing malaria in humans) has thus become a

\footnotetext{
*Correspondence: mapatarr.fidic@gmail.com

${ }^{2}$ Molecular Biology and Immunology Department, Fundación Instituto de Inmunología de Colombia (FIDIC), Carrera 50 No. 26-20, Bogotá,

Colombia

Full list of author information is available at the end of the article
}

challenge for several research groups studying this parasite [7-13].

Unfortunately, maintaining a continuous culture of $P$. vivax in vitro is still difficult, despite different aspects having been studied and modified, i.e. different culture media $[13,14]$, parasite $[9,15]$ and reticulocyte $[7$, 10] sources, added to the different methods for obtaining and enriching invasion target cells [16]. It has only been possible to maintain a culture in vitro for up to 26 months to date, having $<0.1 \%$ parasitaemia [14], which might be due to merozoites (Mrz) losing their ability to re-invade new host cells $[11,13,14,17,18]$.

The forgoing has discouraged research orientated towards knowing in detail the mechanism used by $P$. vivax for specifically invading reticulocytes; consequently, there has been a delay in identifying new molecules, the function they fulfil and their antigenic and immunogenic capability; such information is essential for

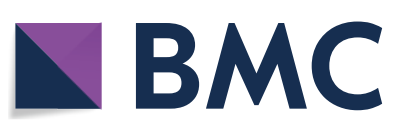

(c) The Author(s) 2018. This article is distributed under the terms of the Creative Commons Attribution 4.0 International License (http://creativecommons.org/licenses/by/4.0/), which permits unrestricted use, distribution, and reproduction in any medium, provided you give appropriate credit to the original author(s) and the source, provide a link to the Creative Commons license, and indicate if changes were made. The Creative Commons Public Domain Dedication waiver (http://creativecommons.org/ publicdomain/zero/1.0/) applies to the data made available in this article, unless otherwise stated. 
selecting specific proteins to be included when developing parasite control methods.

This work has been aimed at reviewing aspects which have been taken into account for standardizing an in vitro $P$. vivax culture and proposes some alternatives which could be considered.

\section{The current state of Plasmodium vivax biology}

Plasmodium vivax is a parasite causing malaria in humans; it has been included on the international health agenda regarding its early eradication, mainly due to the high morbidity rates it causes and its wide geographical distribution [6]. This parasite species displays particular biological characteristics, such as hypnozoite development in the liver and rapid gametocyte formation. Interestingly, the parasite exclusively infects immature erythrocytes (reticulocytes), representing just $1-2 \%$ of total red blood cells (RBC) from adult human peripheral blood. These cells are fragile, have rapid maturation and complex procedures are required for obtaining enriched samples, hence maintaining a $P$. vivax continuous culture in vitro is extremely difficult [16].
The absence of an in vitro culture in $P$. vivax could be considered as "the spoke in the wheel" which has caused a considerable delay (between 5 and 10 years) in executing certain types of studies, such as omic sciences, invasion inhibition and determining adhesin-type ligands, epitopes and antigens [19-21], i.e. compared to those for $P$. falciparum [22-24]. In fact, more than 50 proteins involved in $P$. falciparum binding to and invading target cells have now been described as well as some receptors for them [25-28]. By contrast, only 23 proteins associated with $P$. vivax invasion of reticulocytes have been characterized (using parasites from patients [29-31] and infected animals' samples [32-35]) and few receptors have been studied (Fig. 1). The proteins characterized to date have been tryptophan rich antigens (PvTRAg26.3, PvTRAg33.5, $\quad$ PvTRAg34, $\quad$ PvTRAg35.2, $\quad$ PvTRAg36 (band 3 as receptor) [36], PvTRAg36.6, PvTRAg38 (basigin [37] and band 3 [38] as receptors), PvTRAg40, PvTRAg69.4, PvTRAg74 (band 3 as receptor) [36], rhoptry neck protein 5 (RON5) [39], reticulocyte-binding proteins RBP-1a, RBP-1b [40], RBP-2b (CD71 as receptor) $[41,42]$, erythrocyte binding protein 2 (EBP-2) [43],

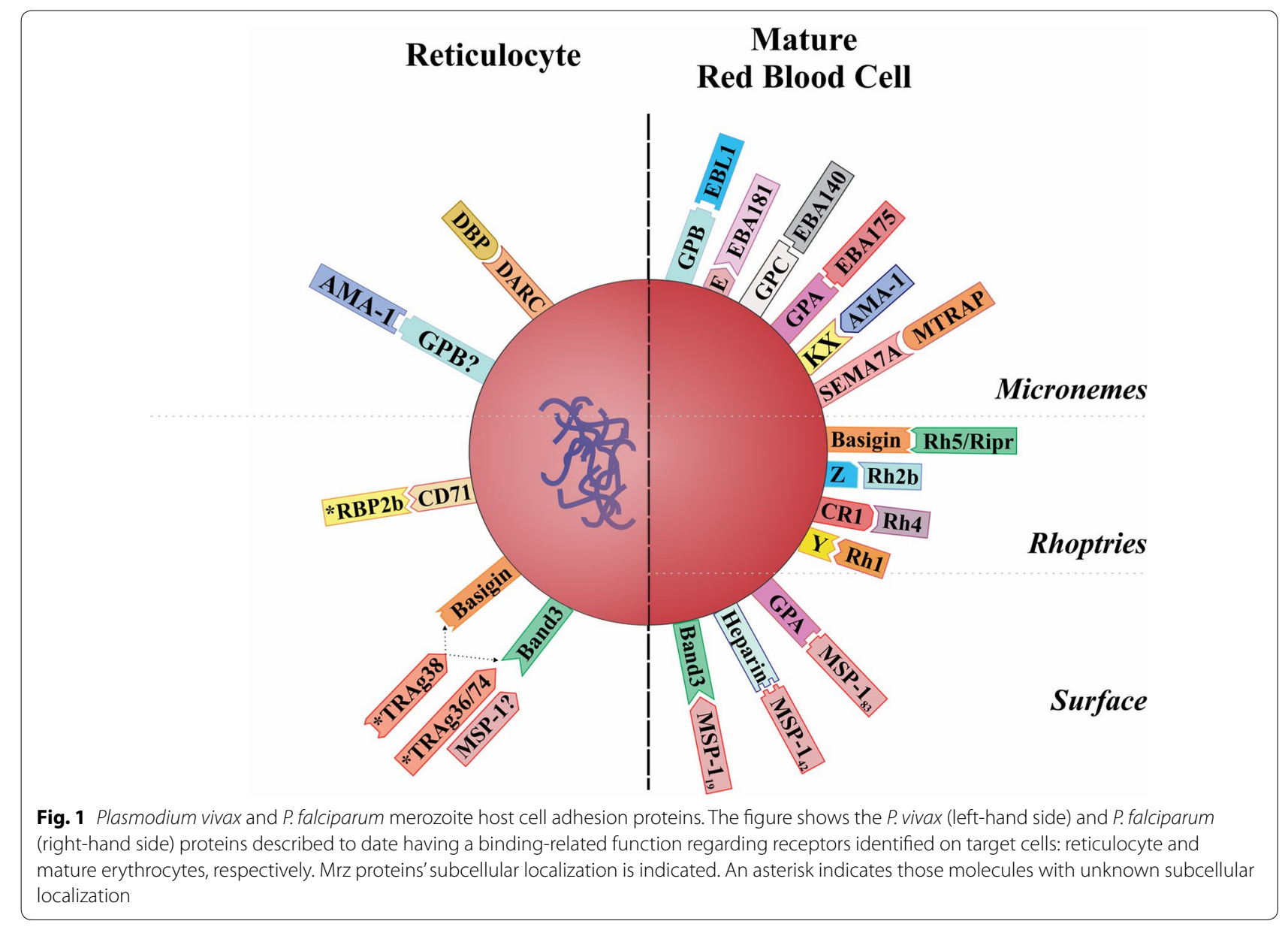


GPI-anchored micronemal antigen (GAMA) [44], reticulocyte binding surface antigen (RBSA) [45], the Duffy binding protein (DBP) (DARC as receptor) $[46,47]$, reticulocyte binding protein 1 (RBP-1) [48], merozoite surface protein 1 (MSP-1) (possible receptor, band 3) [49], apical membrane antigen 1 (AMA-1) (chymotrypsin- and neuraminidase-sensitive receptor, GPB?) [50] and rhoptry neck proteins 2 and 4 (RON2 and RON4) [51].

The small list of characterized ligands and receptors reveals the tremendous challenge faced by researchers considering studying $P$. vivax in terms of continuous propagation to understand different aspects of the parasite's basic biology. In view of this and aimed at making significant advances in clinical and basic research regarding the species, several groups have focused on standardizing a continuous in vitro culture system for $P$. vivax blood stages for which some essential parameters for optimizing parasite growth and development have been determined.

\section{Culturing Plasmodium vivax}

The first reports about culturing malarial Plasmodium date from the beginning of the twentieth century, some techniques being more controversial than others (i.e. parasite culture from infected water and keeping parasites alive in milk for several days) [52]. Although the first successful $P$. vivax in vitro culture was reported in 1912 [53, 54], a base protocol for propagating this parasite species was only established at the end of the 1970s $[4,55,56]$. Since then, one or more of the factors involved in the culture have been modified in various attempts at finding an efficient methodology (Fig. 2). However, it has not been possible to date to maintain a culture, given two main problems: parasitaemia dynamics and the amount of days for maintaining a $P$. vivax in vitro culture. Although is not clear why $P$. vivax Mrz in culture lose their ability to reinvade new host cells, the isolate or parasite strain and target cells may have intrinsic characteristics which can influence $P$. vivax propagation (despite modifications to the culture media). The factors related to maintaining a $P$. vivax in vitro culture (i.e. culture media, parasite and reticulocyte origin) will therefore be analysed.

\section{Culture media and supplements}

Several media and supplement combinations have been tested to ensure the conditions and nutrients required for optimum $P$. vivax growth (Fig. 2). The first reported medias for $P$. vivax in vitro culture were modified Harvard, RPMI-1640, Waymouth's and SCMI 612 supplemented media [4, 53, 57] (Fig. 2); it was seen that only SCMI 612 medium improved parasite viability [58] (Table 1). Other research showed that RPMI 1640 medium supplemented with $\mathrm{MgCl}_{2}$ [59], ascorbic acid, hypoxanthine, vitamin B12, choline and biotin [60] improved parasite maturation. However, in later studies in which RPMI 1640 medium was used [12, 56, 61-65], even in mixture with different compounds and salts $\left(\mathrm{MgSO}_{4}, \mathrm{KH}_{2} \mathrm{PO}_{4}\right.$ and $\left.\mathrm{CaCl}_{2}\right)$ and $50 \% \mathrm{AB}^{+}$human serum, there was no improvement in parasitic density, suggesting that the RPMI 1640 media is not appropriate for $P$. vivax continuous growth and development [65].

McCoy's5A medium has also been routinely used [7, $9-11,13,14,17,18,66,67]$ in combination with various supplements such as D-glucose and L-glutamine, or just with $20 \%$ or $25 \% \mathrm{AB}^{+}$human serum $[9,10,17,18]$. It has been reported that a medium consisting of McCoy's5A supplemented with HEPES, $\mathrm{NaHCO}_{3}$, D-glucose, gentamycin and $50 \% \mathrm{AB}^{+}$human serum maintains parasite density (10 parasites/ $\mu \mathrm{L})$ during the first 5 days of culture. However, such parasite density can be maintained after 5 days using just media supplemented with 25\% $\mathrm{AB}^{+}$human serum [14]. Two compounds improving parasite development in McCoy's5A medium have been reported recently: Albumax II [67] and GlutaMAX [15, 68] (Fig. 2, Table 1). GlutaMAX (L-alanyl-L-glutamine dipeptide) did indeed improve parasite viability, growth and development compared to L-glutamine as this compound does not break down to form toxic by-products, such as ammonia, formed by traditional L-glutamine [15]. This highlighted the fact that $P$. vivax could be very sensitive to the accumulation of waste or toxic products in in vitro conditions.

The use of Dulbecco's Modified Eagle Medium (DMEM) for $P$. vivax culture supplemented with L-glutamine, HEPES and hypoxanthine has been reported recently. Parasitaemia was maintained for 233 days and was ended because of bacterial contamination [69]. The fluctuation in parasitaemia using DMEM was similar to that observed when the parasite has been grown in McCoy's5A medium [14], suggesting that these media (McCoy's 5A and DMEM) are useful for culturing and maintaining parasite maturation and replication in vitro. Future trials should be conducted with McCoy's5A or DMEM medium, supplemented with $25 \%$ human serum (with Glutamax and Albumax) to evaluate whether parasite density can be maintained and/or increased in culture.

\section{Parasite source}

The parasite has been used from two sources for standardizing $P$. vivax in vitro culture, i.e. isolated from humans and from primates (Fig. 2, Table 1). Regardless of the source, it has been observed that keeping the culture in static conditions improves culture parasitaemia [15, 61] as well as depleting white blood cell amount in reticulocyte samples, as leukocytes' phagocytic activity against 


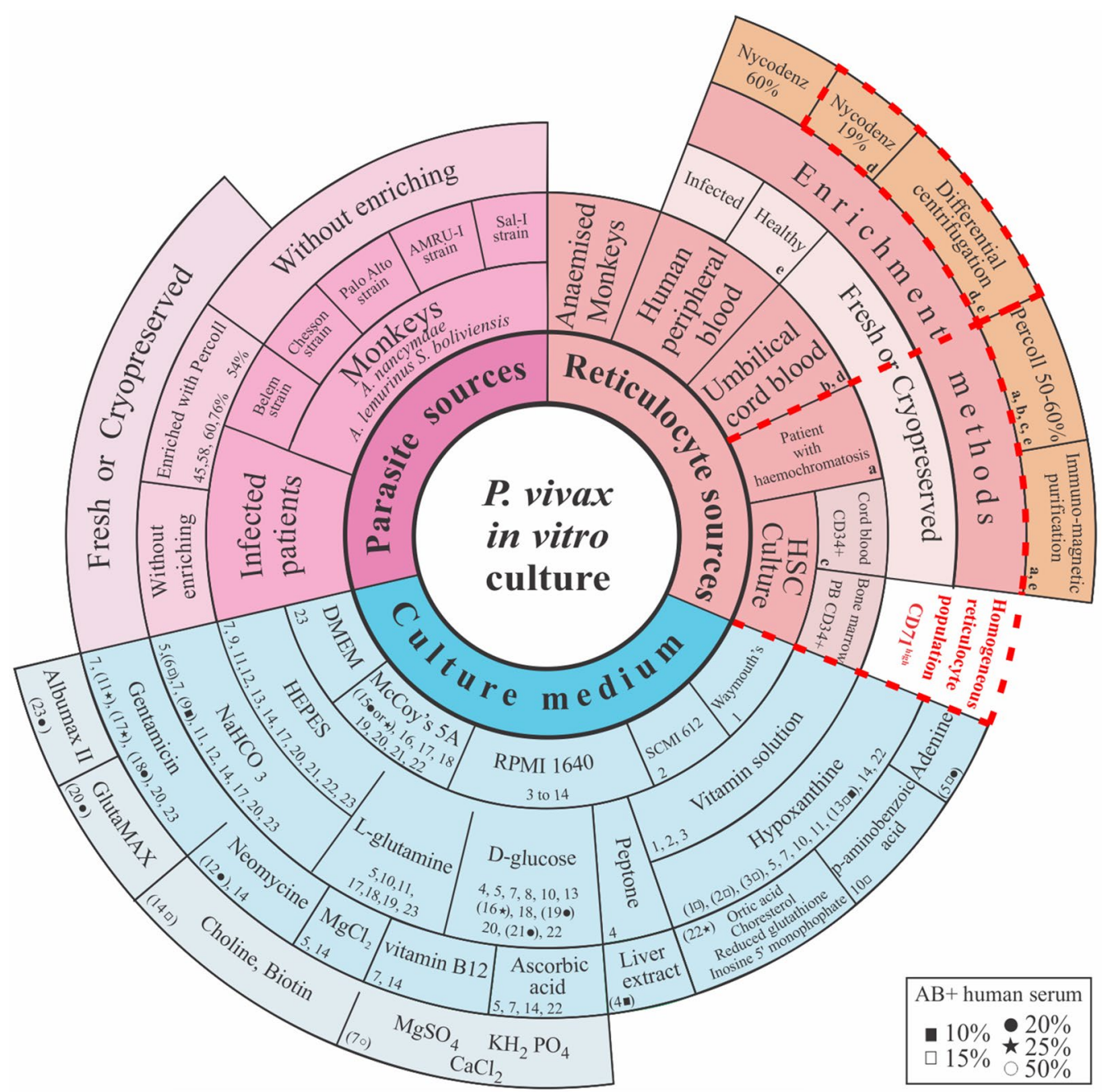

Fig. 2 Conditions evaluated for culturing Plasmodium vivax in vitro. The figure shows modifications made to culture medium, parasite sources and the target cells which have been used in culturing the P. vivax parasite. Each combination evaluated is described in the culture medium section. For example, 14 indicates RPMI 1640 medium, which contains $\mathrm{HEPES}, \mathrm{NaHCO}_{3}$, neomycin, vitamin $\mathrm{B} 12$, hypoxanthine, ascorbic acid, MgCl 2 , biotin, choline and 15\% $\mathrm{AB}^{+}$human serum (box showing typology). The reticulocyte source section lists these cells' enrichment methodology using the letters a-e. Sections of the graphic enclosed by a red discontinuous line indicate the best target cell sources and/or enrichment methods available so far

parasites affects their invasion [53, 61]. Likewise, it has been shown that cryopreservation $[9,18]$ enables maintaining parasite viability and invasive capability when preserved and stored for days $[13,69]$ or even years [9].

Difficulty related to variation in both longevity and parasitaemia has occurred regarding in vitro culture with parasites obtained from humans. For example, it has been reported that different isolates could be maintained in culture for several days: i.e. from 10 or 30 days [12], from 2 to 8 days or up to 85 days (more than 2 months) [10]. An in vitro culture of $3 P$. vivax isolates was recently maintained for more than 1 year (26 months), having $\sim 0.01 \%$ parasitaemia [14]. Other research has shown that culture parasitaemia can increase almost tenfold when using parasites from isolates which were enriched during ring stage by Percoll gradient $[11,68]$. Despite this, the parasite progressively loses its invasion ability, a problem which has not yet been resolved to date. These studies suggested that each $P$. vivax isolate has its own characteristics related to adaptation to in vitro culture and thus their invasion capability, multiplication rate and parasitaemia are variables which must be considered when standardizing a culture for each of them. This hypothesis can be supported by a study by Russell et al., who evaluated umbilical cord blood (UCB) reticulocyte invasion inhibition using $85 P$. vivax clinical isolates. They found that invasion efficiency was constant for each specific isolate but that $85.79 \%$ of the total variance depended on 


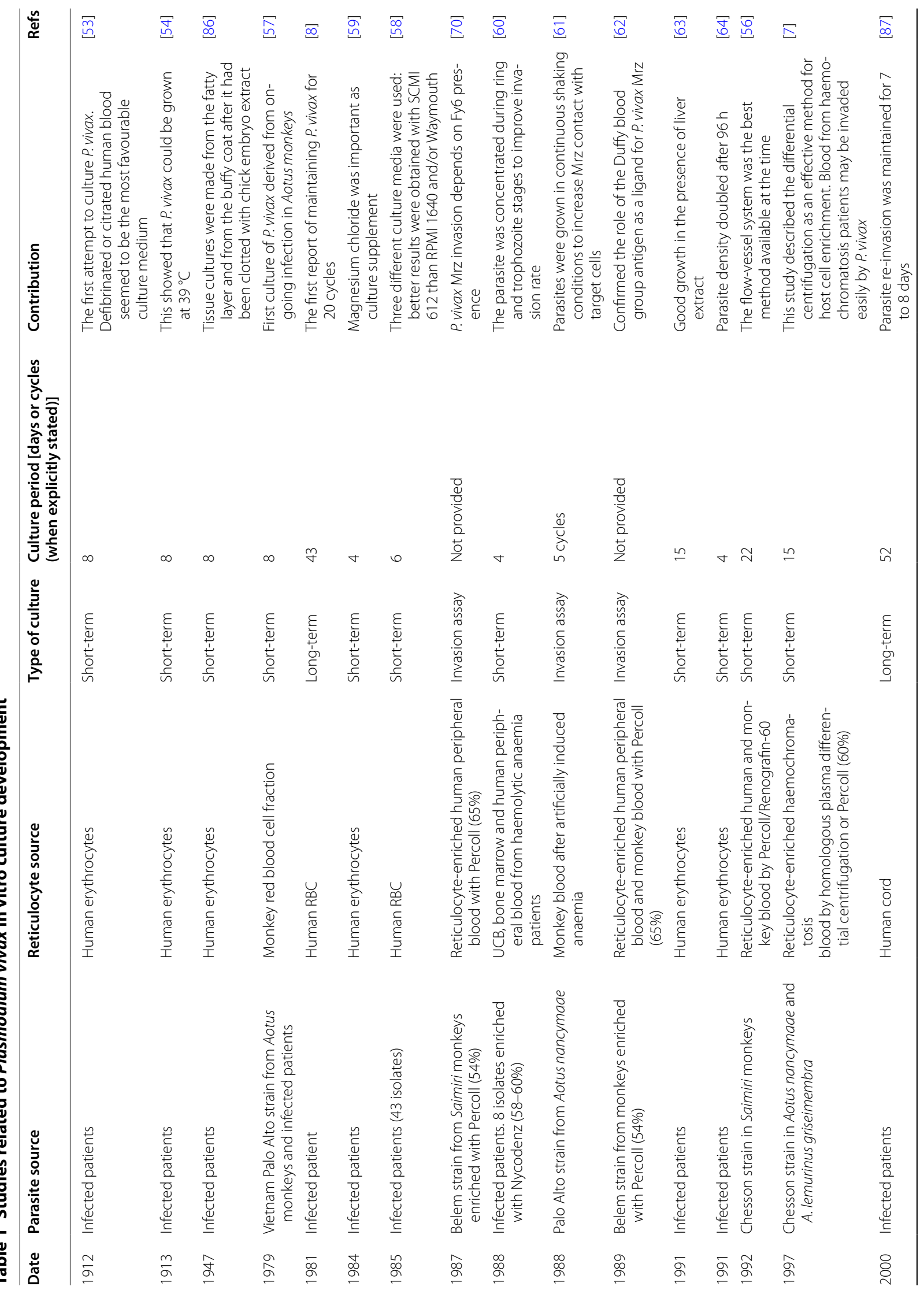




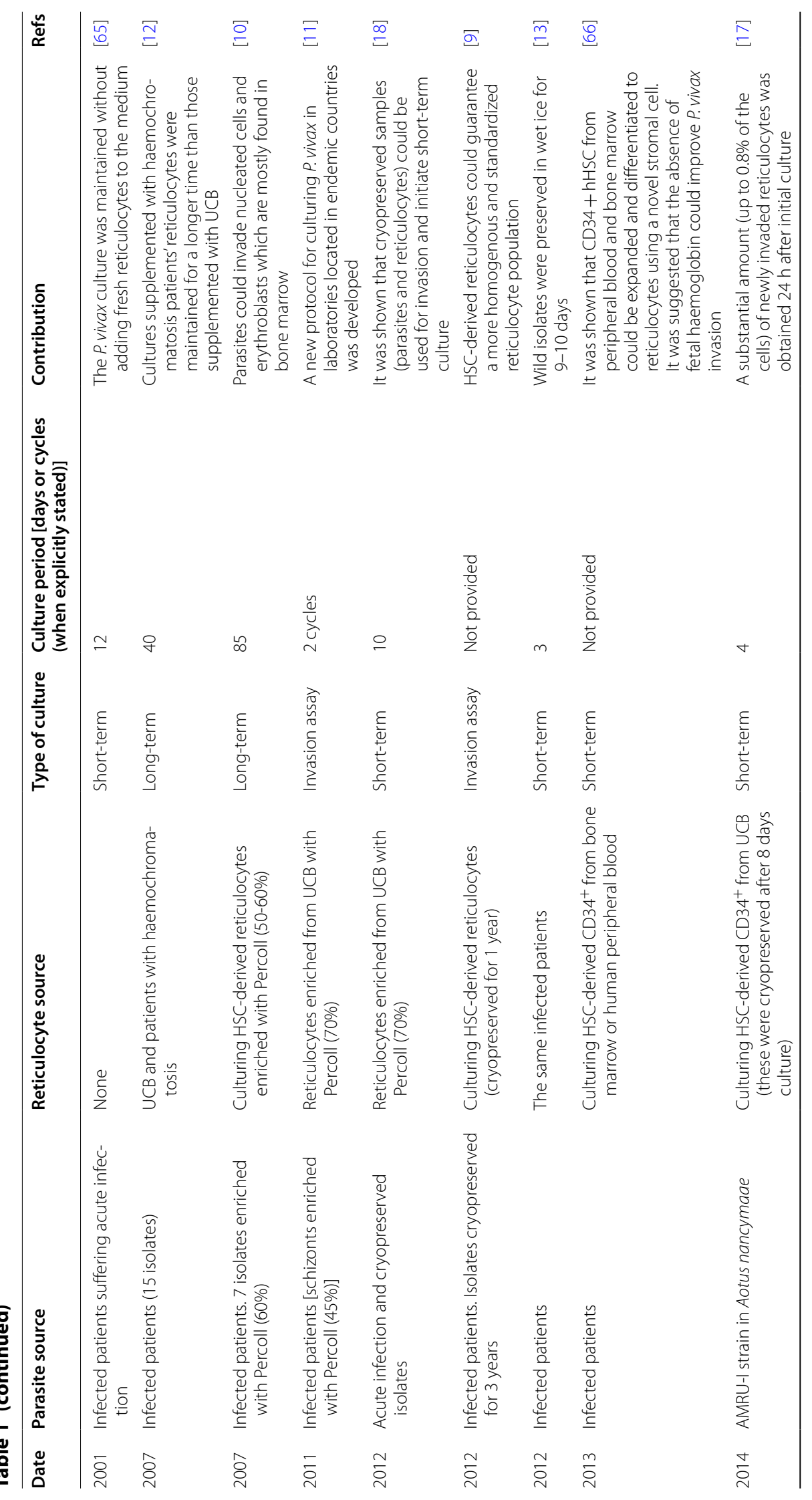




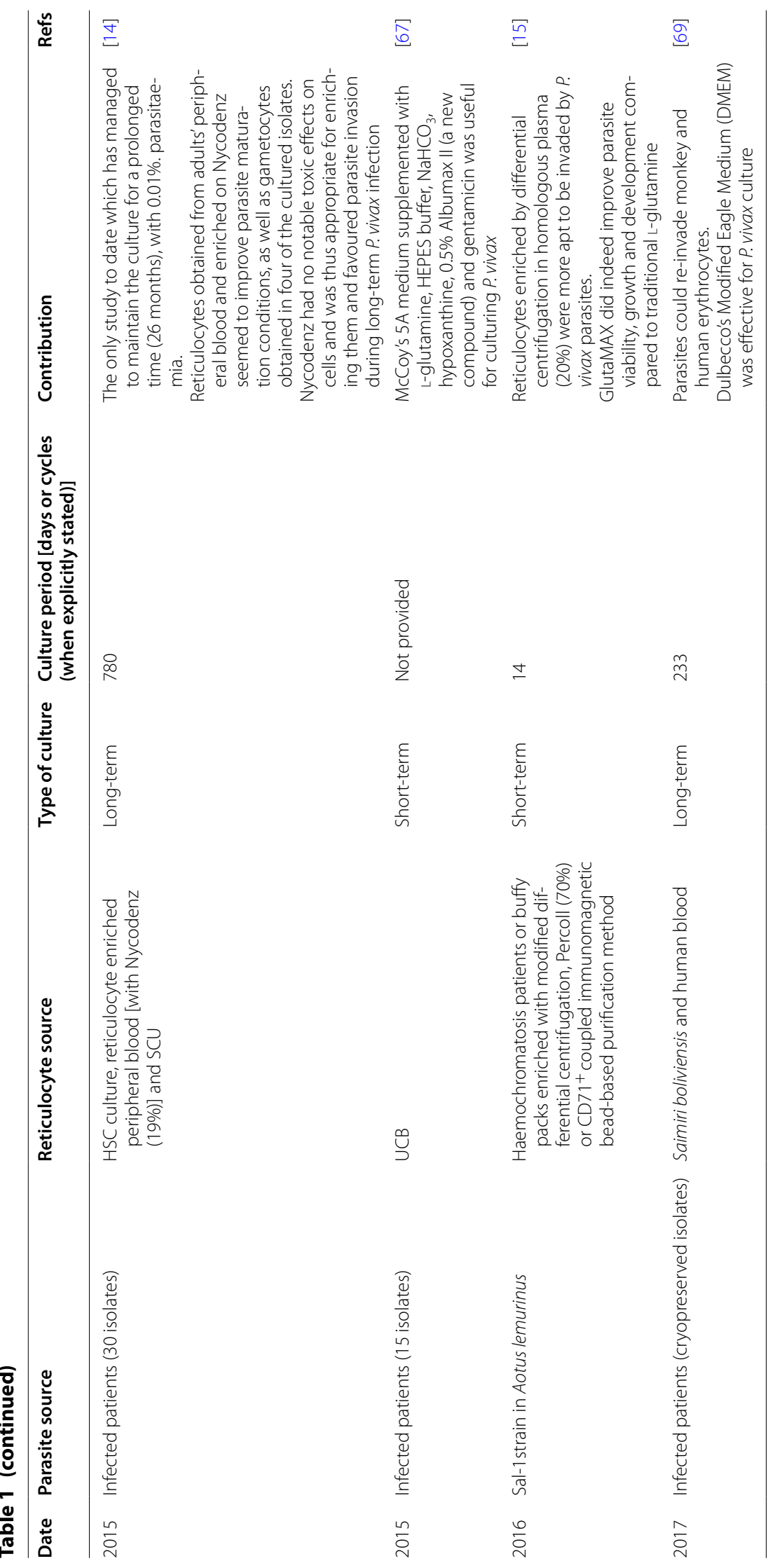


isolate type [11]. Heterogeneity concerning human isolates' invasion efficacy and P. vivax parasitaemia density variation thus makes the methodologies used for culturing the parasite not suitable for studying its biology and further complicates the development of a robust and reliable culture method.

Unlike parasites obtained from humans, primateadapted $P$. vivax strains can be used to start in vitro culture anytime, given their availability. This is why some research groups have worked with several $P$. vivax strains (Fig. 2, Table 1) $[56,57,61,62,70]$ which were able to adapt to invade erythroid cells in vitro (from humans suffering from haemochromatosis $[7,15])$, owl monkey cells [7] and reticulocytes obtained from the maturation of UCB haematopoietic stem cells (HSC) - CD34 ${ }^{+}$[17], cultures reaching $>0.5 \%$ parasitaemia. These results support the notion that monkey-adapted $P$. vivax strains do not lose their capability to invade, regardless of cell source, and therefore, represent a good alternative for establishing a parasite culture.

Considering invasion efficacy variability in cultures from human sources and the great adaptability of strains in monkeys, it can be suggested that the same parasite strain must be used during attempts at standardization to establish the basic and necessary conditions for maintaining a long-term in vitro culture.

\section{Target cell source}

Obtaining reticulocytes for continuous supplementation in culture has been a huge inconvenience since these cells only form 1 to $2 \%$ of human peripheral blood, mature quickly, are fragile and have low viability. UCB (containing 6.9-7.9\% reticulocytes), peripheral blood from humans or splenectomized monkeys, blood from haemochromatosis patients (14-17\% reticulocytes) and HSC (variable reticulocyte percentages) have been used as reticulocyte sources for standardizing an in vitro $P$. vivax parasite culture $[7,12,61]$ (Fig. 2, Table 1). Different techniques such as density gradients (Percoll and Nycodenz), ultra-centrifugation and/or immunomagnetic separation have also been used for obtaining a greater percentage of reticulocytes in culture, Nycodenz being one of the most appropriate compounds as it has had no notable toxic effects on cells [7, 14, 15] (Fig. 2).

Although UCB are a good source of reticulocytes, it has been shown that they do not support the parasite's full development and are easily lysed [61]. Fetal haemoglobin in such erythroid cells apparently produces an inhibitory effect for $P$. vivax growth, equivalent to that reported for $P$. falciparum, which does not grow adequately in erythrocytes containing fetal haemoglobin [71, 72]. The forgoing has been supported since it has been reported that reticulocytes from $\mathrm{CD}_{3} 4^{+}$erythroid progenitors (derived from adult peripheral blood or bone marrow) and from adults' peripheral blood lacking fetal haemoglobin could improve P. vivax invasion [66] regarding maturation and gametocyte production [14]. Whilst these studies have shown that UCB does not seem to be very suitable for standardizing an in vitro $P$. vivax culture, another study has shown that fetal haemoglobin caused no alteration in parasite growth and up to $0.4 \%$ parasitaemia was reached during the first days of culture [11]. Although it is not clear how haemoglobin could alter parasite development, this effect might depend on the reticulocyte's maturation stages (variability) and the availability of these stages in the UCB source.

Another great concern related to using reticulocytes is their rapid maturation. It has been suggested that these target cells can be frozen to provide a reserve and then used for supplementing a culture when required. Different studies have reported that both fresh reticulocytes and freshly thawed reticulocytes were susceptible to invasion by P. vivax Mrz. Interestingly, such susceptibility did not depend on reticulocyte source since they were obtained from UCB [18], haemochromatosis patients (in which the cells were enriched using Percoll gradient [7, 18] or differential centrifugation [7]) and human cord HSCs $[9,17]$ (enriched by Percoll density gradient [10]). Notably, cryopreserved cells which were then thawed had up to $70 \%$ viability and such percentage remained stable compared to that for fresh samples [9].

According to the literature, haemochromatosis patients have been one of the best reticulocyte (fresh or cryopreserved) sources. These reticulocytes, enriched by differential centrifugation in $20 \%$ homologous plasma $[7,15]$, were easily invaded, able to support both parasite growth and invasion [12] and maintain a stable schizont percentage [7]. Although Percoll gradient has been widely used for enriching reticulocytes obtained from haemochromatosis patients (Fig. 2), two studies have reported that cell viability and stability could be affected by damage to or the loss of some membrane receptors which might be essential for $P$. vivax invasion $[7,15]$. Despite this, one of the drawbacks of this target cell source is that haemochromatosis mainly occurs in Caucasians, a type of reticulocyte not normally accessible for researchers outside Europe or North America [14]. Using HSC-derived reticulocytes could guarantee a more homogenous and standardized cell population which would enable obtaining a high reticulocyte concentration $(>20 \%)$ [9], necessary for maintaining $P$. vivax cultures.

Factors such as culture medium, as well as parasite and reticulocyte sources have been revised and possible modifications which could improve parasite development in vitro have been pointed out. However, target cells must be analysed in depth in relation to their intrinsic characteristics enabling the parasite to invade them. 


\section{Reticulocyte receptors: the new molecular keys?}

Taking into account that $P$. vivax Mrz only invade reticulocytes, the next question arises: Which characteristics do reticulocytes have so that $P$. vivax can only invade this type of cell? Immature reticulocytes in bone marrow contain ribonucleic acid (RNA) and undergo different biochemical, biophysical and metabolic changes during their maturation to normocytes within a period of $72 \mathrm{~h}$ [73]. The differences between reticulocytes and normocytes have been studied at molecular level in murine and human models [74-76]. It has been found that the main difference between these two cells is the abundance of their receptors, since more than $60 \%$ of proteins quantified in immature erythrocytes became reduced (from 2 to 100 times) as they matured to normocytes, whilst around 5\% had higher expression levels. Receptors such as transferrin receptor (CD71) on reticulocyte membrane decrease progressively until their total absence in normocytes [77, 78]; this, together with cytoplasmatic RNA (Thiazole Orange stained, TO) concentration, has enabled classifying the reticulocyte population into four groups: Heilmeyer stage I (CD71 $\left.{ }^{\text {high }} \mathrm{TO}^{\text {high }}\right)$, Heilmeyer stages II and III (CD71 $\left.{ }^{\text {low }} \mathrm{TO}^{\text {med}}\right)$ and Heilmeyer stage IV $\left(\mathrm{CD}^{-} \mathrm{TO}^{\text {low }}\right)[73,79]$.

Most $P$. vivax in vitro culture studies have been restricted to using stage III (the first to emerge from bone marrow), stage IV or mature reticulocyte $\left(\mathrm{CD} 71^{-} \mathrm{TO}^{\text {low }}\right)$ populations. Using these two reticulocyte stages and their rapid maturation could provide an explanation for why the parasite loses its infective capability through various replication cycles. This could also explain why less than $1 \%$ parasitaemia has only been achieved in most assays performed to date [12, 14]. In line with the forgoing hypothesis, it has been shown that cryopreserved parasite isolates from patients can infect stage I reticulocytes $\left(\mathrm{CD} 71^{\text {high }} \mathrm{TO}{ }^{\text {high }}\right)$ representing only $0.02 \%$ in total blood [79]. The infected cells mature rapidly and almost completely lose reticular matter $3 \mathrm{~h}$ post-invasion, thereby showing that parasite invasion promotes rapid reticulocyte maturation $[79,80]$. Similar results have been found by Shaw-Saliba et al., when evaluating a culture of Sal-I strain parasites adapted in A. lemurinus monkeys with $\mathrm{CD} 71^{\text {high }}$ reticulocytes. As expected, parasites preferentially invaded stage I reticulocytes $\left(\mathrm{CD} 71^{\text {high }} \mathrm{TO}^{\text {high }}\right)$ and very few managed to invade stage IV reticulocytes $\left(\mathrm{CD} 71^{-} \mathrm{TO}^{\text {low }}\right)$ [15]. Research by Golenda and Udomsangpetch showed that $P$. vivax development and invasion levels were better using haemochromatosis patients' blood; these results also support the previous hypothesis $[7,12]$ as people suffering this type of anaemia produce a larger amount of stage I reticulocytes $\left(\mathrm{CD} 71^{\text {high }} \mathrm{TO}^{\text {high }}\right)$ to balance the decrease of erythrocytes in blood flow [81].
These observations lead to another question: Why can $P$. vivax Mrz only invade the most immature reticulocyte stage? Several studies using different approaches could provide an answer to this question. One such was related to DARC receptor abundance on $\mathrm{CD} 71^{\text {high }} \mathrm{TO}^{\text {high }}$ reticulocytes and conformational changes affecting such receptor enabling the parasite to bind to and invade this type of cell [82]. It has been found that although total DARC protein remains constant throughout reticulocyte maturation there is selective exposure of one DARC amino acid sequence (QLDFEDVWNSSY) by conformational changes before maturation which causes DBP to bind more specifically to $\mathrm{CD} 71^{\text {high }} / \mathrm{TO}^{\text {high }}$ reticulocytes than to other mature reticulocyte or erythrocyte subpopulations [82]. Other studies showing $P$. vivax proteins' preference for binding to $\mathrm{CD} 71^{\text {high }}$ reticulocytes [44, 45 and evidence about RBP-2b binding to CD71 membrane receptor have been published very recently [42]. The above highlights the most immature reticulocyte stage $\left(\mathrm{CD} 71^{\text {high }} \mathrm{TO}^{\text {high }}\right)$ as the molecular key (receptor) which $P$. vivax takes advantage of to invade and replicate within cells. This could suggest that using reticulocytes from bone marrow and/or from patients suffering different types of haemolytic anaemia (i.e. haemochromatosis) could be appropriate for maintaining and developing a continuous in vitro culture system involving $P$. vivax blood stages [83]. However, it would be ethically complicated to work with samples from patients suffering from some type of anaemia, which is why using stage I homogenous reticulocytes $\left(\mathrm{CD} 71^{\text {high }} \mathrm{TO}^{\text {high }}\right)$ obtained from HSC could be a viable alternative.

\section{Others challenges to be faced}

It has been demonstrated that $P$. vivax $\mathrm{DBP}$ binds more to reticulocytes having the $\mathrm{Fya}^{-} / \mathrm{Fyb}^{+}$phenotype [84], which could be an advantage regarding parasite culture.

It would be expected that supplementing cultures with a CD71 ${ }^{\text {high }} \mathrm{TO}^{\text {high }}$ enriched reticulocyte population and having such phenotype should maintain parasitaemia, invasion efficiency and a culture for a long time.

A new challenge today concerns the fact that enough evidence has been amassed to indicate that the parasite can also invade Duffy negative cells ( $\left.\mathrm{Fya}^{-} / \mathrm{Fyb}^{-}\right)$via an asyet-unknown alternative invasion route [85]. This finding suggests two possibilities; first, such $P$. vivax property remains unknown due to this parasite's sub-microscopic and asymptomatic parasitaemia and secondly this could be a new adaptation phenomenon where ligand-receptor interaction routes different to DBP-DARC are acting as survival strategy for propagating cells having the $\mathrm{Fya}^{-} /$ $\mathrm{Fyb}^{-}$phenotype. Studies aimed at ascertaining whether $P$. vivax target cell invasion route is via the RBP2b-CD71 interaction using Duffy negative phenotype $C D 71^{\text {high }}$ 
reticulocytes are in need [42]. Future assays should evaluate whether the aforementioned factors could help standardize a $P$. vivax culture.

Other important considerations include knowing whether the abundance of receptors (as has been showed for DARC and CD71) or remodelling other proteins during reticulocyte maturation (as has been shown for DARC [82]) can have an impact on the development of a $P$. vivax in vitro culture.

This review has described different factors affecting $P$. vivax in vitro culture, ranging from using several strains and isolates to different target cell sources and physicochemical variations. Using the same parasite strain and CD71 ${ }^{\text {high }} \mathrm{TO}^{\text {high }}$ host cells could be a starting point for removing the spoke in the wheel and advance knowledge regarding $P$. vivax biology.

\begin{abstract}
Abbreviations
AMA-1: apical membrane antigen 1; CD71: cluster of differentiation 71 transferrin receptor; DBP: duffy binding protein; DMEM: Iscove's Modified Dulbecco's Medium; EBP-2: erythrocyte binding protein 2; GAMA: GPI-anchored micronemal antigen; RBC: red blood cell; HEPES: (4-(2-hydroxyethyl)-1-piperazineethanesulfonic acid); HSC: haematopoietic stem cell; MSP-1: merozoite surface protein 1; PVTRAg: Plasmodium vivax tryptophan rich antigen; RBP: reticulocyte-binding protein; RBSA: reticulocyte binding surface antigen; RON2: rhoptry neck protein 2; RON4: rhoptry neck protein 4; RON5: rhoptry neck protein 5; RPMI-1640: Roswell Park Memorial Institute medium; UCB: umbilical cord blood; TO: Thiazole Orange.
\end{abstract}

\section{Authors' contributions}

$M B, D A M P$ and GAP analyzed the studies and wrote the initial version of the manuscript. HC and MAP revised and wrote the manuscript. All authors read and approved the final manuscript.

\section{Author details \\ ${ }^{1}$ Receptor-ligand Department, Fundación Instituto de Inmunología de Colombia (FIDIC), Carrera 50 No. 26-20, Bogotá, Colombia. ${ }^{2}$ Molecular Biol- ogy and Immunology Department, Fundación Instituto de Inmunología de Colombia (FIDIC), Carrera 50 No. 26-20, Bogotá, Colombia. ${ }^{3}$ Livestock Sciences Faculty, Universidad de Ciencias Aplicadas y Ambientales (U.D.C.A), Calle 222 No. 55-37, Bogotá, DC, Colombia. ${ }^{4}$ Basic Sciences Department, School of Medicine and Health Sciences, Universidad del Rosario, Carrera 24 No. 63C-69, Bogotá, DC, Colombia.}

\section{Acknowledgements}

We would like to thank Jason Garry for translating this manuscript.

\section{Competing interests}

The authors declare that they have no competing interests.

\section{Availability of data and materials}

All data mentioned in this study are available in the referenced papers.

\section{Consent for publication}

Not applicable.

Ethics approval and consent to participate

Not applicable.

\section{Funding}

MB was financed by the "Formación de Talento Humano de Alto Nivel" Project approved by the "Fondo de Ciencia, Tecnología e Innovación (CTel)" from the "Sistema General de Regalías" (SGR)-BPIN 2013000100103, Gobernación del Tolima and Universidad del Tolima, Colombia. GAP was financed by the
Departamento Administrativo de Ciencias, Tecnología e Innovación (COLCIENCIAS), National Call for Ph.D. Studies in Colombia (No. 567).

\section{Publisher's Note}

Springer Nature remains neutral with regard to jurisdictional claims in published maps and institutional affiliations.

Received: 14 June 2018 Accepted: 13 August 2018

Published online: 20 August 2018

\section{References}

1. Brackett RG, Cole GC, Green TJ, Jacobs RL. In vitro propagation of Plasmodium falciparum for merozoite antigens. Bull World Health Organ. 1979;57(Suppl 1):33-6.

2. Haynes JD, Diggs $C L$, Hines FA, Desjardins RE. Culture of human malaria parasites Plasmodium falciparum. Nature. 1976;263:767-9.

3. Trager W. A new method for intraerythrocytic cultivation of malaria parasites (Plasmodium coatneyi and P. falciparum). J Protozool. 1971;18:239-42.

4. Trager W, Jensen JB. Human malaria parasites in continuous culture. Science. 1976;193:673-5.

5. Schwartz L, Brown GV, Genton B, Moorthy VS. A review of malaria vaccine clinical projects based on the WHO rainbow table. Malar J. 2012;11:11.

6. WHO. World malaria report. Geneva. World Health Organization. 2015;2015:2-3.

7. Golenda CF, Li J, Rosenberg R. Continuous in vitro propagation of the malaria parasite Plasmodium vivax. Proc Natl Acad Sci USA. 1997:94:6786-91.

8. Larrouy G, Magnaval JF, Moro F. Obtaining intraerythrocytic forms of Plasmodium vivax by in vitro culture. C R Seances Acad Sci. 1981; III(292):929-30.

9. Noulin F, Borlon C, van den Eede P, Boel L, Verfaillie CM, D'Alessandro U, Erhart A. Cryopreserved reticulocytes derived from hematopoietic stem cells can be invaded by cryopreserved Plasmodium vivax isolates. PLoS One. 2012;7:e40798.

10. Panichakul T, Sattabongkot J, Chotivanich K, Sirichaisinthop J, Cui L, Udomsangpetch R. Production of erythropoietic cells in vitro for continuous culture of Plasmodium vivax. Int J Parasitol. 2007;37:1551-7.

11. Russell B, Suwanarusk R, Borlon C, Costa FT, Chu CS, Rijken MJ, et al. A reliable ex vivo invasion assay of human reticulocytes by Plasmodium vivax. Blood. 2011;118:e74-81.

12. Udomsangpetch R, Somsri S, Panichakul T, Chotivanich K, Sirichaisinthop J, Yang Z, et al. Short-term in vitro culture of field isolates of Plasmodium vivax using umbilical cord blood. Parasitol Int. 2007;56:65-9.

13. Tantular IS, Pusarawati S, Khin L, Kanbe T, Kimura M, Kido Y, et al. Preservation of wild isolates of human malaria parasites in wet ice and adaptation efficacy to in vitro culture. Trop Med Health. 2012;40:37-45.

14. Roobsoong W, Tharinjaroen CS, Rachaphaew N, Chobson P, Schofield $L$, Cui L, et al. Improvement of culture conditions for long-term in vitro culture of Plasmodium vivax. Malar J. 2015;14:297.

15. Shaw-Saliba K, Thomson-Luque R, Obaldia N 3rd, Nunez M, Dutary S, Lim $\mathrm{C}$, et al. Insights into an optimization of Plasmodium vivax Sal-1 in vitro culture: the Aotus primate model. PLoS Negl Trop Dis. 2016;10:e0004870.

16. Moreno-Perez DA, Ruiz JA, Patarroyo MA. Reticulocytes: Plasmodium vivax target cells. Biol Cell. 2013;105:251-60.

17. Furuya $T$, Sa JM, Chitnis CE, Wellems TE, Stedman TT. Reticulocytes from cryopreserved erythroblasts support Plasmodium vivax infection in vitro. Parasitol Int. 2014;63:278-84.

18. Borlon C, Russell B, Sriprawat K, Suwanarusk R, Erhart A, Renia L, et al. Cryopreserved Plasmodium vivax and cord blood reticulocytes can be used for invasion and short term culture. Int J Parasitol. 2012;42:155-60.

19. Acharya P, Pallavi R, Chandran S, Chakravarti H, Middha S, Acharya J, et al. A glimpse into the clinical proteome of human malaria parasites Plasmodium falciparum and Plasmodium vivax. Proteomics Clin Appl. 2009;3:1314-25.

20. Bozdech Z, Mok S, Hu G, Imwong M, Jaidee A, Russell B, et al. The transcriptome of Plasmodium vivax reveals divergence and diversity of transcriptional regulation in malaria parasites. Proc Natl Acad Sci USA. 2008:105:16290-5. 
21. Carlton JM, Adams JH, Silva JC, Bidwell SL, Lorenzi H, Caler E, et al. Comparative genomics of the neglected human malaria parasite Plasmodium vivax. Nature. 2008:455:757-63.

22. Bozdech Z, Llinas M, Pulliam BL, Wong ED, Zhu J, DeRisi JL. The transcriptome of the intraerythrocytic developmental cycle of Plasmodium falciparum. PLoS Biol. 2003;1:E5.

23. Gardner MJ, Hall N, Fung E, White O, Berriman M, Hyman RW, et al. Genome sequence of the human malaria parasite Plasmodium falciparum. Nature. 2002:419:498-511.

24. Lasonder E, Ishihama Y, Andersen JS, Vermunt AM, Pain A, Sauerwein RW, et al. Analysis of the Plasmodium falciparum proteome by high-accuracy mass spectrometry. Nature. 2002;419:537-42.

25. Cowman AF, Healer J, Marapana D, Marsh K. Malaria: biology and disease. Cell. 2016;167:610-24.

26. Wahlgren M, Goel S, Akhouri RR. Variant surface antigens of Plasmodium falciparum and their roles in severe malaria. Nat Rev Microbiol. 2017;15:479-91

27. Weiss GE, Gilson PR, Taechalertpaisarn T, Tham WH, de Jong NW, Harvey $\mathrm{KL}$, et al. Revealing the sequence and resulting cellular morphology of receptor-ligand interactions during Plasmodium falciparum invasion of erythrocytes. PLoS Pathog. 2015;11:e1004670.

28. Wright GJ, Rayner JC. Plasmodium falciparum erythrocyte invasion: com bining function with immune evasion. PLoS Pathog. 2014;10:e1003943.

29. Cheng Y, Lu F, Tsuboi T, Han ET. Characterization of a novel merozoite surface protein of Plasmodium vivax, Pv41. Acta Trop. 2013;126:222-8.

30. Lee SK, Wang B, Han JH, Nyunt MH, Muh F, Chootong P, et al. Characterization of Pv92, a novel merozoite surface protein of Plasmodium vivax. Korean J Parasitol. 2016;54:385-91.

31. Li J, Ito D, Chen JH, Lu F, Cheng Y, Wang B, et al. Pv12, a 6-Cys antigen of Plasmodium vivax, is localized to the merozoite rhoptry. Parasitol Int. 2012:61:443-9.

32. Collins WE, Skinner JC, Pappaioanou M, Ma NS, Broderson JR, Sutton BB, et al. Infection of Aotus vociferans (karyotype $V$ ) monkeys with different strains of Plasmodium vivax. J Parasitol. 1987;73:536-40.

33. Pico de Coana Y, Rodriguez J, Guerrero E, Barrero C, Rodriguez R, Mendoza $M$, et al. A highly infective Plasmodium vivax strain adapted to Aotus monkeys: quantitative haematological and molecular determinations useful for P. vivax malaria vaccine development. Vaccine. 2003;21:3930-7.

34. Sullivan JS, Morris CL, Richardson BB, Galland GG, Jennings VM, Kendall $J$, et al. Adaptation of the AMRU-1 strain of Plasmodium vivax to Aotus and Saimiri monkeys and to four species of anopheline mosquitoes. J Parasitol. 1999:85:672-7.

35. Sullivan JS, Strobert E, Yang C, Morris CL, Galland GG, Richardson BB, et al. Adaptation of a strain of Plasmodium vivax from India to New World monkeys, chimpanzees, and anopheline mosquitoes. J Parasitol. 2001:87:1398-403.

36. Alam MS, Zeeshan M, Rathore S, Sharma YD. Multiple Plasmodium vivax proteins of Pv-fam-a family interact with human erythrocyte receptor Band 3 and have a role in red cell invasion. Biochem Biophys Res Commun. 2016:478:1211-6.

37. Rathore S, Dass S, Kandari D, Kaur I, Gupta M, Sharma YD. Basigin Interacts with Plasmodium vivax Tryptophan-rich Antigen PvTRAg38 as a second erythrocyte receptor to promote parasite growth. J Biol Chem. 2017;292:462-76.

38. Alam MS, Rathore S, Tyagi RK, Sharma YD. Host-parasite interaction: multiple sites in the Plasmodium vivax tryptophan-rich antigen PVTRAg38 interact with the erythrocyte receptor band 3. FEBS Lett. 2016:590:232-41

39. Arevalo-Pinzon G, Bermudez M, Curtidor H, Patarroyo MA. The Plasmodium vivax rhoptry neck protein 5 is expressed in the apical pole of Plasmodium vivax VCG-1 strain schizonts and binds to human reticulocytes. Malar J. 2015;14:106

40. Han JH, Lee SK, Wang B, Muh F, Nyunt MH, Na S, et al. Identification of a reticulocyte-specific binding domain of Plasmodium vivax reticulocytebinding protein 1 that is homologous to the PfRh4 erythrocyte-binding domain. Sci Rep. 2016;6:26993.

41. Franca CT, He WQ, Gruszczyk J, Lim NT, Lin E, Kiniboro B, et al. Plasmodium vivax reticulocyte binding proteins are key targets of naturally acquired immunity in young Papua New Guinean children. PLoS Negl Trop Dis. 2016;10:e0005014.
42. Gruszczyk J, Kanjee U, Chan LJ, Menant S, Malleret B, Lim NTY, et al. Transferrin receptor 1 is a reticulocyte-specific receptor for Plasmodium vivax. Science. 2018;359:48-55.

43. Ntumngia FB, Thomson-Luque R, Torres Lde M, Gunalan K, Carvalho LH, Adams JH. A novel erythrocyte binding protein of Plasmodium vivax suggests an alternate invasion pathway into Duffy-positive reticulocytes. MBio. 2016;7:e01261.

44. Baquero LA, Moreno-Perez DA, Garzon-Ospina D, Forero-Rodriguez J, Ortiz-Suarez HD, Patarroyo MA. PVGAMA reticulocyte binding activity: predicting conserved functional regions by natural selection analysis. Parasit Vectors. 2017;10:251

45. Moreno-Perez DA, Baquero LA, Chitiva-Ardila DM, Patarroyo MA. Characterising PVRBSA: an exclusive protein from Plasmodium species infecting reticulocytes. Parasit Vectors. 2017;10:243.

46. Batchelor JD, Malpede BM, Omattage NS, DeKoster GT, Henzler-Wildman KA, Tolia NH. Red blood cell invasion by Plasmodium vivax: structural basis for DBP engagement of DARC. PLoS Pathog. 2014;10:e1003869.

47. Ocampo M, Vera R, Eduardo Rodriguez L, Curtidor H, Urquiza M, Suarez J, et al. Plasmodium vivax Duffy binding protein peptides specifically bind to reticulocytes. Peptides. 2002;23:13-22.

48. Urquiza M, Patarroyo MA, Mari V, Ocampo M, Suarez J, Lopez R, et al. Identification and polymorphism of Plasmodium vivax RBP-1 peptides which bind specifically to reticulocytes. Peptides. 2002;23:2265-77.

49. Rodriguez LE, Urquiza M, Ocampo M, Curtidor H, Suarez J, Garcia J, et al. Plasmodium vivax MSP-1 peptides have high specific binding activity to human reticulocytes. Vaccine. 2002;20:1331-9.

50. Arevalo-Pinzon G, Bermudez M, Hernandez D, Curtidor H, Patarroyo MA. Plasmodium vivax ligand-receptor interaction: PVAMA-1 domain I contains the minimal regions for specific interaction with CD71 + reticulocytes. Sci Rep. 2017;7:9616.

51. Bermudez M, Arevalo-Pinzon G, Rubio L, Chaloin O, Muller S, Curtidor H, et al. Receptor-ligand and parasite protein-protein interactions in Plasmodium vivax: Analysing rhoptry neck proteins 2 and 4. Cell Microbiol. 2018:e12835

52. Craig CF. The estivo autumnal (Remittent) malarial fevers. Horace McFarland Company. 1901.

53. Bass CC, Johns FM. The cultivation of malarial plasmodia (Plasmodium vivax and Plasmodium falciparum) in vitro. J Exp Med. 1912;16:567-79.

54. Thomson JG, Thomson D, Fantham HB. The cultivation of one generation of benign tertian malarial parasites (Plasmodium vivax) in vitro, by Bass's method. Ann Trop Med Parasitol. 1913;7:153-64.

55. Trager W, Jensen JB. Cultivation of erythrocytic stages. Bull World Health Organ. 1977;55:363-5.

56. Lanners HN. Prolonged in vitro cultivation of Plasmodium vivax using Trager's continuous-flow method. Parasitol Res. 1992;78:699-701.

57. Siddiqui W. In vitro cultivation of Plasmodium vivax and Plasmodium malariae. Cambridge: Adacemic Press; 1979. p. 279-85.

58. Brockelman CR, Tan-Ariya P, Laovanitch R. Observation on complete schizogony of Plasmodium vivax in vitro. J Protozool. 1985;32:76-80.

59. Brockelman C, Laovanitch R, Kaewkes S. Supportive effects of magnesium chloride on viability of Plasmodium vivax in vitro. J Sci Soc Thailand. 1984;10:109-18.

60. Mons B, Croon JJ, van der Star W, van der Kaay HJ. Erythrocytic schizogony and invasion of Plasmodium vivax in vitro. Int J Parasitol. 1988;18:307-11.

61. Mons B, Collins WE, Skinner JC, van der Star W, Croon JJ, van der Kaay HJ. Plasmodium vivax: in vitro growth and reinvasion in red blood cells of Aotus nancymai. Exp Parasitol. 1988;66:183-8.

62. Barnwell JW, Nichols ME, Rubinstein P. In vitro evaluation of the role of the Duffy blood group in erythrocyte invasion by Plasmodium vivax. J Exp Med. 1989;169:1795-802

63. Sutar NK, Renapurkar DM. Effect of liver extract on growth of Plasmodium vivax in vitro. Indian J Exp Biol. 1991;29:286-7.

64. Zhou WZ, Hu LQ. [Erythrocytic schizogony of Plasmodium vivax under various conditions of in vitro cultivation](in Chinese). Zhongguo Ji Sheng Chong Xue Yu Ji Sheng Chong Bing Za Zhi. 1991;9:258-60.

65. Chotivanich K, Silamut K, Udomsangpetch R, Stepniewska KA, Pukrittayakamee S, Looareesuwan S, et al. Ex-vivo short-term culture and developmental assessment of Plasmodium vivax. Trans R Soc Trop Med Hyg. 2001;95:677-80 
66. Fernandez-Becerra C, Lelievre J, Ferrer M, Anton N, Thomson R, Peligero $C$, et al. Red blood cells derived from peripheral blood and bone marrow CD34(+) human haematopoietic stem cells are permissive to Plasmodium parasites infection. Mem Inst Oswaldo Cruz. 2013;108:801-3.

67. Singh $G$, Urhekar AD, Singh R. In vitro cultivation of Plasmodium vivax using McCoy's medium. Asian J Med Pharm Res. 2015;5:18-21.

68. Rangel GW, Clark MA, Kanjee U, Lim C, Shaw-Saliba K, Menezes MJ, et al. Enhanced ex vivo Plasmodium vivax intraerythrocytic enrichment and maturation for rapid and sensitive parasite growth assays. Antimicrob Agents Chemother. 2018;62:e02519.

69. Mehlotra RK, Blankenship D, Howes RE, Rakotomanga TA, Ramiranirina B, Ramboarina S, et al. Long-term in vitro culture of Plasmodium vivax isolates from Madagascar maintained in Saimiri boliviensis blood. Malar J. 2017;16:442.

70. Nichols ME, Rubinstein P, Barnwell J, de Cordoba RS, Rosenfield RE. A new human Duffy blood group specificity defined by a murine monoclonal antibody Immunogenetics and association with susceptibility to Plasmodium vivax. J Exp Med. 1987;166:776-85.

71. Pasvol G, Weatherall DJ, Wilson RJ. Effects of foetal haemoglobin on susceptibility of red cells to Plasmodium falciparum. Nature. 1977;270:171-3.

72. Pasvol G, Weatherall DJ, Wilson RJ, Smith DH, Gilles HM. Fetal haemoglobin and malaria. Lancet. 1976;1:1269-72.

73. Heilmeyer LWR. Reifungsstadien an Überlebenden Reticulozyten in vitro und ihre Bedeutung für die Schaetzung der täglichen HaemoglobinProduktion in vivo. Ztschr Klin Med. 1932;121:361-79.

74. Liu J, Guo X, Mohandas N, Chasis JA, An X. Membrane remodeling during reticulocyte maturation. Blood. 2010;115:2021-7.

75. Wilson MC, Trakarnsanga K, Heesom KJ, Cogan N, Green C, Toye AM, et al. Comparison of the proteome of adult and cord erythroid cells, and changes in the proteome following reticulocyte maturation. Mol Cell Proteomics. 2016:15:1938-46.

76. Chu TTT, Sinha A, Malleret B, Suwanarusk R, Park JE, Naidu R, et al. Quantitative mass spectrometry of human reticulocytes reveal proteome-wide modifications during maturation. Br J Haematol. 2018;180:118-33.
77. Griffiths RE, Kupzig S, Cogan N, Mankelow TJ, Betin VM, Trakarnsanga K, et al. Maturing reticulocytes internalize plasma membrane in glycophorin A-containing vesicles that fuse with autophagosomes before exocytosis. Blood. 2012;119:6296-306.

78. Koury MJ, Koury ST, Kopsombut P, Bondurant MC. In vitro maturation of nascent reticulocytes to erythrocytes. Blood. 2005;105:2168-74.

79. Malleret B, Li A, Zhang R, Tan KS, Suwanarusk R, Claser C, et al. Plasmodium vivax: restricted tropism and rapid remodeling of CD71-positive reticulocytes. Blood. 2015;125:1314-24.

80. Vryonis G. Observations on the parasitization of erythrocytes by Plasmodium vivax, with special reference to reticulocytes. Am J Epidemiol. 1939;30:41-8

81. Hillman RS. Characteristics of marrow production and reticulocyte maturation in normal man in response to anemia. J Clin Invest. 1969;48:443-53.

82. Ovchynnikova E, Aglialoro F, Bentlage AEH, Vidarsson G, Salinas ND, von Lindern $\mathrm{M}$, et al. DARC extracellular domain remodeling in maturating reticulocytes explains Plasmodium vivax tropism. Blood. 2017;130:1441-4.

83. Martin-Jaular L, Elizalde-Torrent A, Thomson-Luque R, Ferrer M, Segovia JC, Herreros-Aviles E, et al. Reticulocyte-prone malaria parasites predominantly invade CD71 hi immature cells: implications for the development of an in vitro culture for Plasmodium vivax. Malar J. 2013;12:434.

84. King $\mathrm{CL}$, Adams JH, Xianli J, Grimberg BT, McHenry AM, Greenberg LJ, et al. Fy(a)/Fy(b) antigen polymorphism in human erythrocyte Duffy antigen affects susceptibility to Plasmodium vivax malaria. Proc Natl Acad Sci USA. 2011;108:20113-8.

85. Gunalan K, Lo E, Hostetler JB, Yewhalaw D, Mu J, Neafsey DE, et al. Role of Plasmodium vivax Duffy-binding protein 1 in invasion of Duffy-null Africans. Proc Natl Acad Sci USA. 2016;113:6271-6.

86. Dubin IN. Bodies suggesting exoerythrocytic forms of Plasmodium vivax in tissue culture. Proc Soc Exp Biol Med. 1947;65:154-6.

87. Devi C, Pillai C, Subbarao S, Dwivedi SC. Short term in vitro cultivation of erythrocytic stages of Plasmodium vivax. J Parasit Dis. 2000;24:61-6.
Ready to submit your research? Choose BMC and benefit from:

- fast, convenient online submission

- thorough peer review by experienced researchers in your field

- rapid publication on acceptance

- support for research data, including large and complex data types

- gold Open Access which fosters wider collaboration and increased citations

- maximum visibility for your research: over 100M website views per year

At BMC, research is always in progress.

Learn more biomedcentral.com/submissions 